\title{
DISEÑO DE MATERIAL EDUCATIVO PARA LA ENSEÑANZA DE LA CONSERVACIÓN DEL CÓNDOR DE LOS ANDES (Vultur gryphus)
}

\author{
JUAN MANUEL PÉREZ-ZAPATA ${ }^{1}$, CARLOS MOLANO-MONSALVE ${ }^{2}$, JANCY \\ DARLY FLÓREZ-OCHOA ${ }^{1}$, ALEJANDRO RENDÓN-VÁSQUEZ ${ }^{1}$, GLORIA \\ MARCELA FLÓREZ ${ }^{3}$ \\ juanperezzapata@hotmail.com
}

Manizales, 2009-12-01 (Rev. 2010-05-15).

\section{RESUMEN}

En este trabajo se diseñó un material educativo para la enseñanza de la conservación del Cóndor de los Andes (Vultur gryphus) en el Parque Nacional Natural Los Nevados, localizado en la Cordillera Central de Colombia con alturas comprendidas entre 2600 y 5300 metros sobre el nivel del mar. Para la realización de este material se tomó como referentes teóricos el modelo educativo Escuela Nueva, los estándares para la educación en Colombia y los conceptos de lúdica y creatividad. Se realizó una selección de los estándares por grados y por área, y se procedió a estructurar actividades lúdico-creativas de acuerdo con los parámetros establecidos en el modelo educativo Escuela Nueva. El material educativo está compuesto por un texto guía que contiene actividades desde cuatro áreas del conocimiento como lo son las ciencias sociales, las matemáticas, las ciencias naturales y la lengua castellana; y una cartilla que es necesaria para el desarrollo de las actividades que se proponen en el texto guía y contiene la información sobre los aspectos biológicos, ecológicos, literarios y de conservación referentes al cóndor. Se obtuvo un texto guía que contiene seis unidades y 11 guías; y se obtuvo una cartilla con 13 puntos generales sobre el Cóndor de los Andes (Vultur gryphus). El texto guía diseñado ofrece un gran número y variedad de actividades de tipo flexible, lúdicas y creativas que se dinamiza con la cartilla de la especie objeto de conservación. El material educativo diseñado está dirigido a docentes de los centros educativos, facilitadores y promotores ambientales, guías del parque, turistas y a la comunidad en general.

\section{PALABRAS CLAVE:}

Educación ambiental, Escuela Nueva, especies en riesgo, interdisciplinariedad.

\section{DESIGN OF EDUCATIVE MATERIAL FOR TEACHING THE CONSERVATION OF THE ANDEAN CONDOR (Vultur gryphus)}

\section{ABSTRACT}

This project was in charge of designing educative material for teaching the conservation of the Andean Condor (Vultur gryphus) in the Parque Nacional Natural Los Nevados, located in the Central Cordillera of Colombia, with heights ranging between 2600 and $5300 \mathrm{~m}$. As theoretical references, the New School (Escuela Nueva) educational model, the standards for education in Colombia, and the concepts of recreation and creativity were implemented. A selection of standards by degree and by area was carried out, as well as organizing the activities in accordance with the parameters established in the New School educational model. The educative material consists of a guide text which contains 
activities from four areas of knowledge such as social sciences, mathematics, natural sciences and the Spanish language, and a handbook necessary for the development of the activities proposed in the guide text and contains information about biological, ecological, literature and conservational aspects concerning the condor. The result was a guide text that contains six units and 11 guides, and a handbook with 13 general points on the Andean Condor (Vultur gryphus). The guide text offers a great number and variety of flexible, recreational and creative activities that is invigorated with the condor handbook. The educational material designed is aimed at school teachers, facilitators and environmental promoters, park guides, tourists and the general community.

KEY WORDS: environmental education, New School, endangered species, interdisciplinary approach.

\section{INTRODUCCIÓN}

Desde la década de los años sesenta la educación ambiental fue asumida como un principio fundamental para la conservación y protección del medio ambiente, al ser evidente los perjuicios en los ecosistemas causados por la sociedad (Gonzales, 2003). A partir de la década de los setenta se han venido unificando esfuerzos en torno a la temática ambiental por organizaciones como las Naciones Unidas (UN), el Programa de las Naciones Unidas para el Medio Ambiente (PNUMA) y la Organización de las Naciones Unidas para la Educación, la Ciencia y la Cultura (UNESCO), las cuales recomiendan establecer un Programa Internacional de Educación Ambiental (PIEA) que aborde desde la interdisciplinaridad, la educación formal y no formal, cubriendo todos los niveles de enseñanza y dirigido a todos los sectores, jóvenes y adultos (PNUMA, 1981; UNESCO-PNUMA, 1985).

La interdisciplinaridad o transversalidad es propia de lo pedagógico y plantea la integración de las disciplinas, tomando un tema en común, abordable desde la especificidad de cada una de ellas (Gonzales, 2003). Al ser lo ambiental, complejo e integrador de aspectos físico-naturales y necesario para concienciar, comprender y apreciar la relación individuo, sociedad y ambiente (MolanoMonsalve, 2004), permite transversalizar las áreas del conocimiento, llegándose a hablar de ambientalización de la educación, un concepto que busca integrar todas las disciplinas, saberes y prácticas en torno a temas ambientales y propone el trabajo conjunto entre educación formal, no formal e informal (Noguera et al., 2006).

Según el Ministerio de Educación Nacional (2002) en Colombia, para conservar el ambiente, es necesario un sistema educativo dinámico, que tenga una clara relación con la realidad y con las necesidades de la sociedad. En este sentido existen varios lineamientos y conceptos con los cuales se puede trabajar de manera integrada, los cuales son: el modelo pedagógico Escuela Nueva que está dirigido a comunidades rurales y urbanas de bajos recursos económicos y que pretende utilizar un método de aprendizaje interactivo, donde se articulen temas intersectoriales tales como el medio ambiente; los estándares para la educación en Colombia expedidos por el Ministerio de Educación Nacional (MEN) que especifican aquello que deben saber y ser capaces de hacer según área y grado los estudiantes de educación preescolar, básica primaria, básica secundaria y media vocacional; la lúdica que aborda diferentes temas desde la óptica del juego, permitiendo dimensionar desde otras vivencias la realidad, y la creatividad definida como la habilidad puesta de manifiesto en cada individuo con el 
propósito de instrumentar mejoras en un entorno determinado; involucrando las actividades psicomotoras, cognitivas y afectivas (Penagos \& Aluni, 2000; Ospina \& Gómez, 2001).

Todos estos aspectos anteriormente mencionados nos abren la posibilidad de proponer un trabajo conjunto entre educación formal, no formal e informal partiendo desde la situación de amenaza en la que se encuentran muchas de las especies que hacen parte de nuestra biodiversidad. Entre estas especies se encuentra el Cóndor de los Andes (Vultur gryphus), el cual ha disminuido su población debido al desarrollo económico de la zona andina que ha ocasionado pérdida de hábitat y disminución de la oferta alimentaria (Rengifo et al., 2002). El Cóndor de los Andes es considerada como una "Especie Sombrilla" lo cual le da una enorme importancia para la protección de áreas y conservación de la biodiversidad; además de la posibilidad de asociarla con la generación de servicios ambientales, acciones de conservación de ecosistemas y mejora de la calidad de vida de comunidades. Son varias las acciones que se han llevado a cabo para la conservación de esta especie, las cuales incluyen: reintroducción, monitoreos, educación ambiental y capacitación de personal técnico, líderes y comunidades que tienen relación con el cóndor (Corpocaldas, 2000; Feliciano \& Arcila, 2000; Rodríguez-Mahecha \& Orozco, 2002; Corpocaldas, 2005). Sin embargo, estos importantes esfuerzos no han sido transversalizados en las diferentes áreas del conocimiento.

Este trabajo tiene como objetivo el diseño de material educativo para la enseñanza de la conservación del Cóndor de los Andes (Vultur gryphus), y que en su conjunto integre de manera dinámica diferentes áreas del conocimiento. Los resultados de este trabajo hacen parte de una primera fase del proyecto titulado: "Diseño de una estrategia metodológica para la enseñanza de la educación ambiental con especies animales objeto de conservación en el Parque Nacional Natural Los Nevados". Dicho proyecto ha sido propuesto por la Fundación Gestión Colombia - Manizales.

\section{MATERIALES Y MÉTODOS}

Para el diseño del texto guía se revisaron los estándares para la educación en Colombia de las áreas: lengua castellana, matemáticas, ciencias sociales y ciencias naturales para los grados primero a tercero y cuarto a quinto de la educación básica, y se tomaron aquellos que por su flexibilidad podían ser aplicados a la temática ambiental para una especie objeto de conservación. Se revisaron de igual forma los ejes articuladores, los logros y los indicadores de logros dentro de los cuales se enmarca cada estándar. Finalmente, se procedió a realizar las actividades lúdico-creativas de acuerdo con los parámetros establecidos en el modelo Escuela Nueva, los cuales obedecen al siguiente esquema:

A. Actividad Básica "Partamos de tus ideas" En estas actividades se indaga sobre las ideas o conocimientos previos de los estudiantes y tienen como objetivo desarrollar destrezas, actitudes y habilidades.

B. Cuento pedagógico "Aprendamos algo nuevo" En estas actividades se realizan lecturas a partir de una cartilla, dinámicas de lectura grupal o individual, trabajo con nuevas palabras y comprensión de lectura. 
C. Actividad práctica "Usemos lo que aprendemos" Son actividades individuales o en pequeños grupos en las cuales se busca el desarrollo de actitudes de compañerismo, solidaridad y participación.

D. Actividad libre "Lo que aprendemos y nuestra vida" Estas actividades buscan ampliar conocimientos, interacción con su grupo social y familiar propiciándose así el desarrollo socio-afectivo.

Para la creación de las actividades se tuvieron en cuenta de igual forma los dominios de la conducta: Cognoscitivo (Piensa), Afectivo (Siente) o Sicomotora (Actúa).

Para el diseño de la cartilla se tomó como especie al Cóndor de los Andes (Vultur gryphus); se tuvo en cuenta para su contenido los aspectos biológicos, ecológicos, literarios y de conservación referentes al cóndor.

\section{RESULTADOS Y DISCUSIÓN}

Se obtuvo un texto guía que contiene seis unidades y 11 guías.

Para el área de lengua castellana se diseñaron tres unidades y siete guías. Éstas siguen a continuación con su respectivo título:

\section{UNIDAD 1}

Guía 1 Conociendo la especie según nuestros ancestros.

Guía 2 Si la especie nos contara sus historias.

Guía 3 Aquello que sentimos por los seres con los cuales compartimos la naturaleza.

\section{UNIDAD 2}

Guía 4 La naturaleza nos cuenta sus historias.

Guía 5 Ayudo a la naturaleza a contar sus historias.

\section{UNIDAD 3}

Guía 6 El conocimiento científico acerca de la especie.

Guía 7 La especie en las noticias.

Para el área de ciencias naturales se diseñó una unidad y dos guías:

\section{UNIDAD 1}

Guía 1 Clasifiquemos los seres de la naturaleza.

Guía 2 Dónde encontramos los seres vivos y cómo debemos cuidar nuestro ambiente.

Para el área de matemáticas se diseñó una unidad y una guía:

\section{UNIDAD 1}

Guía 1 Entornos y vida. Relación natura-cultura.

Para el área de ciencias sociales se diseñó una unidad y una guía: 


\section{UNIDAD 1}

Guía 1 Mi comunidad, mi comunidad ancestral y sus relaciones con la especie.

Al final de cada una de las unidades, el facilitador encontrará, además de la literatura utilizada para ilustrar los temas de las guías, fuentes de consulta adicional a las cuales podrá remitirse para complementar y crear nuevas actividades.

Se obtuvo de igual forma una cartilla con 13 puntos generales sobre el Cóndor de los Andes (Vultur gryphus) que toma como contenido los siguientes aspectos:

I. Narraciones Acerca de la Especie (Mito, Leyenda, Fábula, Cuento, Historieta, Poema, Obra de teatro de títeres, Artículo científico y Notas periodísticas).

II. Descripción General de la Especie (Taxonomía, Anatomía, Alimentación, Reproducción, Conducta, Otros nombres que recibe la especie y Ficha biológica de la especie).

III. Cómo es el medio donde vive la especie, sus adaptaciones y sus relaciones con otros seres vivos.

IV. Relación de la especie con el hombre y su importancia.

V. Características extraordinarias de la especie.

VI. Distribución (mapas actuales, ancestrales, paisajes naturales, paisajes culturales).

VII. Acciones emprendidas para su conservación en Colombia y en el PNN Los Nevados.

VIII. Amenazas y su estado actual de conservación en Colombia y en el PNN Los Nevados.

IX. Propuestas de conservación.

X. ¿Qué podemos hacer para proteger y conservar a esta especie?

XI. Glosario ambiental.

XII. Consulta en línea.

XIII. Bibliografía.

Al igual que los trabajos realizados por Velázquez et al. (2007) y Velázquez y Otero (2006), para el diseño del material educativo se empleó la metodología de enseñanza activa, los estándares para la calidad de la educación en Colombia y la lúdica y la creatividad; sin embargo, el material educativo diseñado difiere en varios puntos con el aquí presentado. Los mismos autores parten desde temas propios de la educación ambiental como la conservación, el cuidado, el deterioro del ambiente, la biodiversidad, etc., hacia áreas del conocimiento como las ciencias sociales, las matemáticas, la educación artística y la lengua castellana, lo cual es diferente a lo realizado en este trabajo ya que se parte desde la situación actual y la información detallada acerca de una especie objeto de conservación hacia diferentes áreas del conocimiento como las ciencias sociales, las matemáticas, la lengua castellana y la educación ambiental. Por otra parte en el trabajo arriba citado, tanto los docentes como los estudiantes emplean el mismo texto didáctico, no así en este trabajo ya que el texto guía y la cartilla diseñados son empleados por el facilitador o docente y el participante del encuentro ambiental, respectivamente.

\section{CONCLUSIONES}

Este material educativo para la conservación del Cóndor de los Andes (Vultur gryphus) está elaborado para que intérpretes ambientales, facilitadores y docentes desarrollen un proyecto ambiental en los ámbitos formal e informal, 
llevando a cabo jornadas teórico-prácticas para el proceso de aprendizaje en las niñas, los niños y jóvenes, con carácter escalonado y cualificado que contribuya al desarrollo de conciencia ambiental positiva, de valores, actitudes y comportamientos frente a su entorno, y que a su vez favorezca al conocimiento y conservación de esta especie.

El Texto guía podrá ser aplicado a cualquier especie objeto de conservación en cualquier Parque Nacional Natural, de la que se tenga una cartilla elaborada con los parámetros descritos en este trabajo y puede ser dinamizado por facilitadores ambientales o por docentes desde su área de interés.

\section{REFERENCIAS BIBLIOGRÁFICAS}

- Corpocaldas. (2000, Oct.). Proyecto Conservación y Protección del Cóndor Andino en el Parque Nacional Natural Los Nevados. Gestión Ambiental 5. Manizales, Caldas.

- . (2005). El Cóndor Vuelve a Casa. Los cóndores del Parque Nacional Natural Los Nevados. Manizales, Caldas.

- Feliciano, O y Arcila, E. (2000). El Cóndor vuelve a casa. Ministerio del Medio Ambiente, unidad coordinadora - Fundación Renaser, Santafé de Bogotá.

- Gonzales, A. (2003). Tranversalidad - interdisciplina. Obtenido el 3 de noviembre de 2009, desde http://www.cladead.com/cursos/MEDAM/MEDAM000003/transversalid ad.htm\#_ftn2

- Ministerio de Educación Nacional, Colombia. (1998). Ciencias Naturales y Educación Ambiental. Lineamientos Curriculares. Bogotá D. C. - Colombia: El Ministerio.

- (2002). Estándares para la Excelencia en la Educación. Bogotá, D. C. - Colombia. Ed Creamos Alternativas Ltda.

- Molano-Monsalve, C. (2004, Ene.). Desarrollo de la Conciencia Ambiental por medio de la Lúdica, Una propuesta desde la Educación Ambiental para el Desarrollo Rural. Revista Luna Azul, No. 18. Disponible en: http://lunazul.ucaldas.edu.co/index.php?option=com_content\&task=b logsection \&id=10\&/temid $=30$

- Noguera P, Pineda J, Echeverri, J, Sánchez, I, Contreras C, Valencia C. (2006). Propuesta Plan Decenal de Ambientalización de la Educación 2005-2014. Manizales: Universidad Nacional - Secretaría de Educación de Caldas - Corpocaldas. Inédito.

- Organización de las Naciones Unidas para la Educación, la Ciencia y la Cultura y el Programa de las Naciones Unidas para el Medio Ambiente, UNESCO-PNUMA. (1985). Educación ambiental: hacia una pedagogía basada en la resolución de problemas. No. 15.

- Ospina, B. y Gómez, Diana. (2001). Lúdica como Estrategia Metodológica para el Cambio de Actitud de los Estudiantes Frente al Área de Ciencias Naturales. Tesis no publicada, Universidad de Caldas, Facultad de Ciencias Exactas y Naturales, Programa de Biología y Química. Manizales.

- Penagos, J. C. y Aluni R. (2000). Creatividad, una aproximación. Revista Psicológica, edición especial "Creatividad". Obtenido el 11 de noviembre de 2007, desde http://homepage.mac.com/penagoscorzo/creatividad. 2000 
- Programa de las Naciones Unidas para el Medio Ambiente (PNUMA). (1981). Resolución 9/20/A, mayo de 1981. Obtenido el 3 de noviembre de 2009, desde http://www.pnuma.org/educamb/

- Rengifo, L.M., A.M. Franco-Maya, J.D. Amaya-Espinel, G.H. Kattan \& B. López-Lanús (Eds.). (2002). Libro Rojo de Aves de Colombia. Serie de Libros Rojos de Especies Amenazadas de Colombia. Instituto de Investigaciones Biológicas Alexander von Humboldt (IAvH) y Ministerio de Ambiente, Vivienda y Desarrollo Territorial (MAVDT). Bogotá, D.C. Colombia.

- Rodríguez-Mahecha, J.V., R.H. Orozco. (2002). Vultur gryphus. En: Rengifo, L.M., A.M. Franco-Maya, J.D. Amaya-Espinel, G.H. Kattan, B. López-Lanús (Eds.), Libro rojo de Aves de Colombia. Serie Libros Rojos de Especies Amenazadas de Colombia. Instituto de Investigación de Recursos Biológicos Alexander von Humboldt (IAvH) y Ministerio del Medio Ambiente. Bogotá, D.C. - Colombia.

- Velázquez, J., Flórez, G., Moreno, J., Sabogal, P., Manrique, S. (2007). Diseño, elaboración y validación de textos didácticos en educación ambiental para básica primaria, básica secundaria y media vocacional. Texto Didáctico de Educación Ambiental Grado 6. Soñando un Planeta. Manizales, Colombia: Editorial Manigraf.

- Velázquez, J. y Otero, E. (2006). Diseño, elaboración y validación de textos didácticos en educación ambiental para básica primaria, básica secundaria y media vocacional. Texto Didáctico de Educación Ambiental Grado 5. Soñando un planeta. Manizales, Colombia: Editorial Manigraf.

1. Maestría en Ciencias Veterinarias, Universidad de Caldas, Manizales, Colombia.

2. Fundación Gestión Colombia, Manizales, Colombia.

3. Departamento de Estudios Educativos, Universidad de Caldas, Manizales, Colombia. 